\title{
Physical Layer Security for Massive MIMO: An Overview on Passive Eavesdropping and Active Attacks
}

\author{
Dževdan Kapetanović, Gan Zheng and Fredrik Rusek
}

\begin{abstract}
This article discusses opportunities and challenges of physical layer security integration in massive multiple-input multiple-output (MaMIMO) systems. Specifically, we first show that MaMIMO itself is robust against passive eavesdropping attacks. We then review a pilot contamination scheme which actively attacks the channel estimation process. This pilot contamination attack is not only dramatically reducing the achievable secrecy capacity but is also difficult to detect. We proceed by reviewing some methods from literature that detect active attacks on MaMIMO. The last part of the paper surveys the open research problems that we believe are the most important to address in the future and give a few promising directions of research to solve them.
\end{abstract}

Dževdan Kapetanović did this work while at the Interdisciplinary Center for Security, Reliability and Trust (SnT), University of Luxembourg, Luxembourg, E-mail: dzevdan.kapetanovic@gmail.com.

Gan Zheng is with School of Computer Science and Electronic Engineering, University of Essex, UK, E-mail: ganzheng@essex.ac.uk.

Fredrik Rusek is with Dept. of Electrical and Information Technology, Lund University, Lund, Sweden, E-mail: fredrik.rusek@eit.Ith.se. 


\section{INTRODUCTION}

During the recent past the field of massive multiple-input multiple-output (MaMIMO) systems has quickly emerged as one of the most promising techniques to boost the system throughput of emerging and future communication systems. In MaMIMO, the vision is to equip the base station (BS) with an antenna array comprising 100+ antenna elements and a large number of independent transceiver chains. An impressive amount of research on the topic has been conducted, and MaMIMO will be integrated in the upcoming 5G standard [1], [2]. The advantages of MaMIMO are manifold, and to give a few we mention (i) An array gain corresponding to the number of BS antenna elements, (ii) A channel hardening effect, rendering stable and predictable channel conditions to users, (iii) Nearly orthogonal channels from the BS to the users, (iv) Simple signal processing at both the BS and at the users.

Another advantage of MaMIMO, yet not widely recognized, is that the potential of physical layer security (PLS) against passive eavesdropping attacks is increased dramatically. PLS is based on the following result: Consider a Gaussian wiretap channel [3] where a BS communicates with a legitime user (LU) in the presence of a passive eavesdropper (ED). Assume that the Shannon capacities from the $\mathrm{BS}$ to the LU and the ED are $\mathcal{C}_{\mathrm{LU}}$ and $\mathcal{C}_{\mathrm{ED}}$, respectively. Then, the secrecy capacity between the BS and the $\mathrm{LU}$ is $\mathcal{C}_{\mathrm{SC}}=\max \left\{\mathcal{C}_{\mathrm{LU}}-\mathcal{C}_{\mathrm{ED}}, 0\right\}$. This rate can be transmitted reliably and securely without any use of a formal crypto system. In conventional MIMO systems, the two capacities $\mathcal{C}_{\mathrm{LU}}$ and $\mathcal{C}_{\mathrm{ED}}$ are of similar order of magnitude, rendering a fairly small secrecy capacity. With MaMIMO and passive eavesdropping, the situation changes dramatically. With standard time-division duplex (TDD) mode MaMIMO operations, and due to (i) - (iii) above, the received signal power at the LU is several orders of magnitude larger than the received signal power at the ED. This generates a situation where the secrecy capacity is nearly the full capacity to the LU, i.e., $\mathcal{C}_{\mathrm{SC}} \approx \mathcal{C}_{\mathrm{LU}}$ [4]. Altogether, MaMIMO enables excellent PLS, without any extra effort.

There are of course countermeasures that can be taken by the ED. First of all, it could place itself physically close to the LU so that the channels to the LU and the ED are highly correlated. In this case, (iii) does not longer hold true, and the secrecy capacity may be compromised. Another effective strategy that the ED can adopt is to exploit the weakness of the channel estimation phase in MaMIMO. By switching from a passive to an active mode, the ED can pretend to be the LU and send a pilot sequence of his own - this is the so called pilot contamination attack. The BS will then beamform signal power to the ED instead of the LU. To detect such a stealthy attack is challenging since there is a normal amount of pilot contamination already present in MaMIMO systems. 
Active ED attacks are by no means unique to MaMIMO. On the contrary, jamming the BS is a well researched attack in conventional MIMO. See for example [5], [6] for two recent papers where the ED attacks the channel estimation phase. The ED can also combine passive eavesdropping and active jamming attacks. The strategies for countering such an attack in conventional MIMO channels are discussed in [7]. A game-theoretic approach is taken in [8] to deal with combination of passive and active attacks. However, none of the aforementioned papers explicitly dealt with MaMIMO systems and the inefficiency of passive eavesdropping in MaMIMO was not observed.

Although MaMIMO has received huge attention, the existing literature on the combination of PLS and MaMIMO is scarce. This paper will survey the opportunities that MaMIMO may bring for making PLS a reality, as well as discussing problems that must be tackled in the future. The paper sets off by discussing the benefits that MaMIMO brings to PLS in the presence of a passive ED. We then highlight that active attacks are more likely to occur for MaMIMO. Three detection methods to deal with active attacks are then briefly reviewed. We then outline important open problems to address. The paper is concluded by a discussion of a few promising directions of future research. We believe that there are many interesting aspects of PLS integration in MaMIMO to be researched and hope that this survey will attract the attention of the research community to this exciting and open field.

\section{Passive And Active Eavesdropping Attacks}

For conceptual simplicity, we consider a single cell with an $M$-antenna BS, one single-antenna LU, and one single-antenna ED. The uplink channels from the LU and the ED to the BS are denoted as $\mathrm{g}_{\mathrm{LU}}$ and $\mathbf{g}_{\mathrm{ED}}$, respectively. We assume a TDD system where channel reciprocity holds, and the corresponding downlink channels are $\mathbf{g}_{\mathrm{LU}}^{\mathrm{T}}$ and $\mathbf{g}_{\mathrm{ED}}^{\mathrm{T}}$ where $(\cdot)^{\mathrm{T}}$ denotes transpose operation. Standard TDD MaMIMO involves two phases: (i) The LU transmits a training symbol to the BS in the uplink, and (ii) Relying on channel reciprocity, the BS performs channel estimation and beamforms the signal to the $\mathrm{LU}$ in the downlink using the uplink channel estimation $\hat{\mathrm{g}}_{\mathrm{U}}$ with proper scaling.

The ED aims to overhear the communication from the BS to the LU while at the same time being undetected. To this end, the ED can launch either a passive attack or an active attack, which will be reviewed below.

\section{A. Passive Eavesdropping Attack}

Let us now discuss the passive attack within a MaMIMO context as shown in Fig. 1. The key observation here is that the presence of a passive ED is not at all affecting the beamforming at the BS and has an 
negligible effect on the secrecy capacity. Intuitively, this is because MaMIMO has the capability to focus the transmission energy in the direction of the LU. This implies that the received signal strength at the ED is much less than that at the LU. In Fig. 1, the resulting ergodic capacities $\mathcal{C}_{\mathrm{LU}}$ and $\mathcal{C}_{\mathrm{ED}}$ are shown as functions of the number of BS antennas $M$ with perfect channel estimation. It is assumed that $\mathbf{g}_{\mathrm{LU}}$ and $\mathbf{g}_{\mathrm{ED}}$ are independent and identically distributed (i.i.d) complex Gaussian vectors with equal mean powers, and the BS transmit power is normalized to $0 \mathrm{~dB}$.

As can be seen from Fig. 1, the ED's capacity remains the same as $M$ increases; this is so since the BS does not beamform in the ED's direction. The capacity to the LU, however, is greatly increased for large values of $M$. In other words, the secrecy capacity is about half of the LU capacity with conventional MIMO $(M \approx 2-8)$, while it constitutes more than $85 \%$ of $\mathcal{C}_{\mathrm{LU}}$ already at $M=100$. From this simple example, we can see that MaMIMO has excellent potential for integration of PLS.

\section{B. An active attack on the channel estimation}

The resilience of MaMIMO against the passive attack is based on the assumption that the uplink channel estimation $\hat{\mathrm{g}}_{\mathrm{LU}}$ is independent of the ED's channel $\mathbf{g}_{\mathrm{ED}}$. This motivates the ED to design active attacks on the channel estimation process to influence the BS's beamforming design. Next we describe such an attack based on the pilot contamination scheme in [10].

As illustrated in Fig. 2, during the uplink channel estimation, the LU transmits a pilot symbol to the BS. At the same time, the ED launches the attack by sending another pilot symbol. In the worst case the ED is synchronized with the legitimate transmission and this is possible by overhearing the signaling exchange between the BS and the LU.

The consequence of this attack, if left undetected, is that the promising PLS benefits of MaMIMO are lost. The difference from the passive attack is that the channel estimate $\hat{\mathrm{g}}_{\mathrm{LU}}$ becomes correlated with the ED's channel $\mathbf{g}_{\mathrm{ED}}$, and consequently the equivalent channel for the ED also improves as $M$ increases. Even worse, if the ED uses higher training power, it dominates the training phase and the secrecy capacity may become zero.

For the same settings as in Fig. 1, we plot the ergodic channel capacities and the ergodic secrecy capacity in Fig. 2, The signal sent by the ED is $10 \mathrm{~dB}$ weaker than the training signal sent by the LU. As can be seen, both the ED's and the LU's channel capacities increase with M, however, the secrecy capacity remains constant for $M>50$.

Although the situation is similar to the well-known pilot contamination problem in multi-cell systems [9], a notable difference is that the ED is out of control and therefore existing schemes for reducing pilot 
contamination in MaMIMO cannot be applied. In the remainder of this paper we survey recent progress to detect the active attack and discuss possible future directions of research.

\section{Detection Schemes}

As described in Section II-B, detection of an active ED is crucial for secure MaMIMO communication. Now we will present arguments that show how peculiar and different ED detection is in MaMIMO systems.

Consider a detection scheme applied by the BS during uplink packet transmission, that is based on successful packet reception. In systems such as LTE and WLAN, the uplink packet contains channel estimation pilots. If the ED attacks these pilots, one could argue that this would result in decoding errors and thus packet loss (due to a bad channel estimate). Indeed, this is what happens in conventional MIMO systems with few antennas (depending on the robustness of the used modulation and coding scheme, of course). Therefore, the BS would suspect presence of strong interference, either coming from an ED or another user, and could take actions based on this. However, in MaMIMO, the erroneous channel estimate does not typically result in a decoding error, assuming that ED's channel is uncorrelated with LU's channel. Hence, in contrast to a conventional MIMO system, a successful packet reception does not imply absence of an ED in a MaMIMO system. As we have argued, if this erroneous channel estimate is left undetected and arises from an active ED, it can have a detrimental effect on the secrecy capacity if used in the subsequent downlink phase. Hence, the importance of effective detection schemes during channel estimation.

An appealing and conceptually simple detection strategy that can be used during channel estimation, which we will also argue against, is to let the BS first estimate the mean power or large scale fading $\beta$ of the estimated uplink channel vector $\hat{\mathrm{g}}_{\mathrm{LU}}$, i.e., $\beta=\mathbb{E}\left[\left\|\hat{\mathrm{g}}_{\mathrm{LU}}\right\|^{2}\right]$. The value $\beta$ changes slowly over time/frequency so that a good estimation of it is feasible. The instantaneous received energy of a pilot observation at the $\mathrm{BS}$ converges to $\beta P+N_{0}$ as $M$ grows large, where $P$ is the power of the pilot symbol and $N_{0}$ is the noise density at the BS. Thus, if $\beta$ and $N_{0}$ are known, the BS can compare the instantaneous received energy with $\beta P+N_{0}$. In the absence of an active ED the two quantities are close to each other, while an active ED is detected if the instantaneous received energy is much larger than $\beta P+N_{0}$.

Unfortunately, there is a simple countermeasure that the ED can take. Since the value of $\beta$ changes slowly, the ED can adapt its transmit power in order to sabotage the estimation of $\beta$ at the BS. The ED starts transmitting at low power, and increases the power over several coherence intervals of $\beta$. The 
ED is thereby emulating the natural change of the channel propagation environment, and the BS can not distinguish the increased received power from a natural channel quality improvement. The lesson learned from this example is that detection methods should not solely depend on the large scale fading parameter $\beta$ and should preferably work without the knowledge of $\beta$. This section will describe two different schemes (one with two flavours) that can effectively detect such an attack in MaMIMO systems without the knowledge of $\beta$. Both schemes share two important features: simplicity and effectiveness, which are due to MaMIMO. Although ED's presence during the channel estimation phase removes the expected MaMIMO gain in secrecy capacity, as demonstrated in section II-B, MaMIMO instead enables simple and effective schemes for detection of the ED.

\section{A. Detection Scheme 1: LU transmits random pilot symbols}

This scheme exploits controlled randomness in transmitting "random" pilots to detect an active ED. As first proposed in [11], the LU transmits a sequence of random phase-shift keying (PSK) symbols, which forms the key to detecting the ED at the BS. Below we discuss two variations of this scheme.

1) Scheme 1a: Two pilot symbols $p_{1}$ and $p_{2}$ transmitted by the LU are chosen independently from an $N$-PSK constellation. The BS receives the two pilot signals $\mathbf{y}_{1}$ and $\mathbf{y}_{2}$. The BS now forms the detection statistic $z$ as the phase of $\mathbf{y}_{1}^{\mathrm{H}} \mathbf{y}_{2}$, where $(.)^{H}$ is conjugate transpose. Three scenarios are possible:

1) The ED is absent during both transmissions. In this case, the phase of $z$ can be shown to converge to a phase of a valid PSK symbol as the number of antennas grows large.

2) The ED is present in both slots. As the number of antennas grow large, the phase of $z$ converges with probability $1-1 / N$ to a number that is not a valid phase of an $N$-PSK symbol.

3) The ED is present in only one of the slots. In this case, $z$ still converges to a valid PSK phase. However, the received power at the BS will be biased towards a larger value during the slot where the ED is present. Hence, one can form the ratio $q=\left\|\mathbf{y}_{1}\right\|^{2} /\left\|\mathbf{y}_{2}\right\|^{2}$. If $q<\gamma_{1}$ or $q>\gamma_{2}$, for some thresholds $\gamma_{1}, \gamma_{2}$, then it is decided that the ED is present.

From 2), we know that as the antenna number $M$ grows large, the probability of detection converges to $1-1 / N$. Thus, it can be made arbitrarily close to 1 by increasing the alphabet size $N$ while the false alarm probability converges to 0 due to 1 ). In order to use a large value of $N, M$ must be quite large $(M>200)$. Note that this simple scheme is effective due to MaMIMO, which provides convergence of the scalar product to different values depending on ED's presence. Moreover, for large $M$, this scheme is robust to knowledge of the noise power $N_{0}$. Namely, the noise is averaged out in the scalar product between the received signals when $M$ is large. 
2) Scheme $1 b$ : The second random pilot scheme provides improvements to the above scheme in case of three or more observations. Given $L$ observations $\mathbf{y}_{1}, \ldots, \mathbf{y}_{L}$, form the matrices

$$
\begin{aligned}
\mathbf{Y} & =\left[\mathbf{y}_{1}, \ldots, \mathbf{y}_{L}\right], \\
\mathbf{R} & =\frac{\mathbf{Y}^{H} \mathbf{Y}}{M}-N_{0} \mathbf{I},
\end{aligned}
$$

where $\mathbf{I}$ is an $L \times L$ identity matrix. When $M$ becomes large, $\mathbf{R}$ converges to a rank-one matrix if ED is absent, otherwise it converges to a full rank matrix with probability $1-1 / N$. Based on this observation, the following detection rule is applied: if $\lambda_{1}\{\mathbf{R}\} / \lambda_{2}\{\mathbf{R}\}$ is greater than some threshold, ED is absent; otherwise it is present, where $\lambda_{1}\{\mathbf{R}\}$ and $\lambda_{2}\{\mathbf{R}\}$ are the largest and the second largest eigenvalues of R.

We will later see in section III-C that this scheme provides significant performance enhancement with only four observations. The scheme also takes care of the case when the ED is not present in all $L$ slots. Again, noteworthy is the simplicity of this scheme, which is due to MaMIMO since it enables convergence of $\mathbf{R}$ to matrices of different rank depending on ED's presence. Although performing significantly better than scheme 1a, scheme $1 \mathrm{~b}$ requires a good estimate of $N_{0}$, since it is used to construct $\mathbf{R}$.

\section{B. Detection Scheme 2: Cooperative Detection Scheme}

The above random detection incurs the overhead of transmitting additional random sequences. We next briefly introduce a detection method that does not transmit additional pilot symbols [12].

As illustrated in Fig. 4, upon receiving the training signal from the LU, the BS can apply a beamformer based on the received signal, and then transmit a pilot to the LU using the same beamformer. The beamformer is constructed in such a way that the received sample at the LU in the absence of an active ED equals an agreed value (between the $\mathrm{BS}$ and the $\mathrm{LU}$ ) after a scaling with $1 / M$. For simplicity, this agreed value can be taken as 1 in our discussion. In the case that there is an active ED, the LU will observe a much smaller quantity, and this forms our basis to detect the presence of the ED.

As before, simple beamforming is effective due to MaMIMO. Similar to the random pilot scheme $1 \mathrm{~b}$, the cooperative scheme also requires a decent estimate of $N_{0}$ for proper performance.

\section{Comparison of Detection Schemes}

We discuss the pros and cons of the introduced detection schemes and show a comparison of detection performance in Fig. 5 with 200 antennas at the BS. The channel vectors $\mathbf{g}_{\mathrm{LU}}$ and $\mathbf{g}_{\mathrm{ED}}$ are independent with each element drawn from a complex Gaussian distribution with mean 0 and variance 1 . The pilot 
powers from the LU and the ED are also equal. For the random pilot schemes, we use a QPSK alphabet. For the cooperative scheme, it is assumed that the noise power at the BS equals the noise power at the LU.

As shown in Fig. 5, the cooperative scheme performs best at moderate to high SNRs. The dotted curve illustrates the performance of random pilot scheme 1a. Its performance is inferior to the cooperative scheme at moderate to high SNRs, but significantly better at low SNRs. The dashed curve represents random pilot scheme $1 \mathrm{~b}$ with four received slots, which exhibits very good performance compared to the random pilot scheme with two slots.

As depicted in Fig. 5, the biggest advantage of the cooperative scheme is its detection performance during the two exchange intervals. The drawback with the cooperative scheme is that the ED can cause problems during the whole frame exchange. For example, the ED might be very close to the LU, and thus contaminate any packets that the LU receives from the BS. The noise power at the LU will therefore be significantly higher than the noise power at the BS, causing a degradation in the detection performance at the LU.

The advantage of the random pilot schemes are their robustness to jamming of the LU by the ED, since the detection is performed at the BS. If the ED would increase its power toward the BS, it would instead result in yet easier detection of the ED. However, as illustrated in Fig. 5, the random pilot schemes requires more than two observations of the received signal in order to have a performance comparable or better than the cooperative scheme.

\section{Further Discussions And Future Directions}

In this section, we discuss limitations of the solutions introduced above and give promising future directions.

\section{A. Limitations}

The reason why only the active attack is effective in MaMIMO is that the channels to the LU and the ED are assumed to be largely uncorrelated. If the channels were correlated, or in the worst case the same, then there is no need for the ED to be active. This means that if the ED can position itself such that its channel to the BS is highly correlated with the channel from the LU to the BS, then the ED can be passive. Furthermore, in line-of-sight scenarios the beamforming at the BS is directional. If the ED is at the very same angle-of-departure from the BS, the ED will receive a highly correlated signal although it may not be very close. We point out that this problem is alleviated in 3D beamforming scenarios. 
These considerations are important to take into account in further work to delineate suitable scenarios for combining PLS with MaMIMO.

\section{B. Detection of Active Attacks in Multi-user and Multi-cell Systems}

We have so far been dealing solely with a single cell and a single user. The case of a multi-user scenario does not alter the situation much as the users can be allocated orthogonal resources for transmitting training symbols. Thus, there is virtually no interaction among users during the training phase. A multicell scenario, on the other hand, brings about radical changes. This is so since even without any active ED, the received signal at the BS corresponding to the LU's training signal is being interfered with by LUs from other cells; this is the so called pilot contamination problem of massive MIMO [2]. Consequently, in a multi-cell scenario, the problem of detecting the presence of an active ED changes into the much more challenging problem of distinguishing an active ED from LUs in other cells. In a multi-cell scenario, the detection methods from Section III will all fail since they will detect the presence of the other cells' LUs, but are not sophisticated enough to distinguish these from an active ED.

The multi-cell active ED detection problem is a wide open research problem as, to the best of the authors' knowledge, there are no attempts in the existing literature to deal with it. Although not much is known, we briefly introduce two potential ways forward that can be pursued in future research.

1) Cooperative BSs: Cooperative BSs is a technology that has already found its way into the LTE standard, both in the form of the Coordinated Multipoint (CoMP) and as the less complex Network Assisted Interference Cancellation and Suppression (NAICS). In these architectures, the BSs of different cells are connected via a backhaul network and exchange information. Consequently, there is a possibility to let the BSs jointly estimate the level of LU-induced pilot contamination. Even better, the cooperative BSs can jointly try to minimize the pilot contamination in the system. Then the detection methods in Section III can be applied verbatim. If the detection methods identify a suspiciously high level of pilot contamination, then the transmission can be terminated. In the case that there was in fact no active ED, but the LU-induced pilot contamination level was unusually high, the terminated transmission is not a major problem as the transmission is not very efficient whenever the pilot contamination is high.

2) Methods based on radio propagation characteristics: In a multi-cell case, pilot contamination is caused by LUs from other cells. To be effective, the ED should arguably be located within the serving cell. The radio propagation characteristics between users and a MaMIMO BS are today well understood, and a reasonable working assumption is that the statistical properties of incoming radio waves to the BS from LUs far away are different from those coming from a potential ED that is located much closer to 
the BS. This is so since signals from users in other cells are typically being reflected by a few dominant objects in the vicinity of the users, and these objects may have line-of-sight to the serving cell BS. Thus, the radio waves' angle-of-arrivals (AoAs) for users in other cells may be limited to a few possibilities, while the signal from a close by ED may reach the BS via interacting objects in the vicinity of the BS. This opens up a possibility to differentiate between pilot contamination from LUs and pilot contamination from an active ED.

3) The angle-of-arrival database for location-aware users: A particularly interesting direction of research may be to combine the outlined approach in Section IV-B2 with the results from [13]. In [13], the authors discuss possibilities of systems where LUs can obtain position estimates of themselves. If the LUs report their positions and signal-strengths to a central node, then a database of signal strength as function of physical position can be constructed. In MaMIMO, this idea can be extended and utilized to detect active EDs. Rather than building a database of signal-strengths, a database can contain the AoAs to the BS from LUs at certain positions. The detection of an active ED would then comprise the following steps: (i) the LU reports his position to the BS , (ii) the LU transmits a training symbol, (iii) the BS requests the AoAs for the particular user position from the database, (iv) if the measured AoAs for the pilot observation does not match the input from the database, there is an active ED. So far, the authors are not aware of any attempt in the literature to build an AoA database, and future research is needed to investigate the feasibility of this approach.

Again, if the ED can get physically close to the LU the measured AoAs may match those in the database even in the presence of an active ED . However, recent measurement campaigns [14] with a 128 port antenna array and where 8 users are located within a five-meter diameter circle show that the radio propagation characteristics are sufficiently different for the 8 users (both in line-of-sight and non-line-of-sight) so that they can all be spatially separated. This indicates that a MaMIMO system may very well be capable of distinguishing an ED from a LU even when they are physically close.

\section{Detection using Learning Mechanism}

Machine learning is a powerful tool that comes natural to mind for detecting active EDs. Machine learning comes in two forms: supervised and unsupervised learning. In supervised learning one must guarantee that there is no active ED present when training the machine. When this is not possible to guarantee unsupervised learning should be used. However, we can foresee a number of problems that arise that must be dealt with. Firstly, a fairly large amount of training data must be used in machine learning, which may cause an unacceptably high overhead. In MaMIMO this is a particular severe problem as the 
amount of training may grow with the antenna number. Secondly, the mobility of users is a problem since the channel quality and characteristics may vary dramatically over time and space.

Alternatively, device dependent radio-metrics such as frequency and phase shift differences of radio signals can be used as unique fingerprints to detect active EDs [15]. This method is channel-invariant, but extracting the features is a complicated task. More efforts need to be made to resolve these problems in order to use machine learning tools for detecting active EDs.

\section{CONCLUSions}

MaMIMO has shown great potential to boost system capacity but the combination of PLS and MaMIMO is not well understood yet. In this article, we have reviewed both attacks and detection schemes. In particular, we have shown that although passive eavesdropping has little effect on the secrecy capacity, an active attack on the channel estimation phase is harmful. We have presented three detection schemes that can effectively identify the active attack. The detection of active attacks in multi-cell and multi-user systems is especially relevant to the applications of MaMIMO - but is challenging and not much is known today. Some promising research directions to this end have been discussed in the paper.

\section{REFERENCES}

[1] E. G. Larsson, O. Edfors, F. Tufvesson and T. L. Marzetta, "Massive MIMO for next genera- tion wireless systems," IEEE Commun. Mag., pp. 186-195, Feb. 2014.

[2] F. Rusek, D. Persson, B. K. Lau, E. G. Larsson, T. L. Marzetta, O. Edfors, and F. Tufvesson, "Scaling up MIMO,” IEEE Signal Process. Mag., vol. 30, no. 1, pp. 40-60, Jan. 2013.

[3] A. Khisti and G. W. Wornell, "Secure transmission with multiple antennas: The MISOME wiretap channel," IEEE Trans. Inf. Theory, vol. 56, no. 7, pp. 3088-3104, July 2010.

[4] J. Zhu, R. Schober, and V. Bhargava, "Secure transmission in multi-Cell massive MIMO systems," accepted in IEEE Trans. Wireless Commun. Available online: http://arxiv.org/abs/1405.7161

[5] R. Miller and W. Trappe, "On the vulnerabilities of CSI in MIMO wireless communication systems," IEEE Trans. Mobile Comput., vol. 11, no. 8, pp. 1386-1398, Aug. 2012.

[6] S. Sodagari and T. C. Clancy, "On singularity attacks in MIMO channels," Wiley Transactions on Emerging Telecommunication Technologies, May 2013.

[7] A. Mukherjee and A. L. Swindlehurst, "Optimal strategies for countering dual-threat jamming/eavesdropping-capable adversaries in MIMO channels,“ IEEE Military Communications Conference (MILCOM), pp. 1695-1700, 2010.

[8] Q. Zhu, W. Saad, Z. Han, H. V. Poor, and T. Basar, "Eavesdropping and jamming in next-generation wireless networks: a game theoretic approach,“ IEEE Military Communications Conference (MILCOM), pp. 119-124, 2011.

[9] J. Jose, A. Ashikhmin, T. L. Marzetta, and S. Vishwanath, "Pilot contamination and precoding in multi-cell TDD systems," IEEE Trans. Wireless Commun., vol. 10, no. 8, pp. 2640-2651, Aug. 2011. 
[10] X. Zhou, B. Maham and A. Hjørungnes, "Pilot Contamination for Active Eavesdropping," IEEE Trans. Wireless Commun., vol. 11, no. 3, pp. 903-907, Mar. 2012.

[11] D. Kapetanovic, G. Zheng, K. K. Wong, and B. Ottersten, "Detecting Pilot Contamination Using Random Training and Massive MIMO,” IEEE Personal, Indoor and Mobile Radio Communications (PIMRC), London, UK, Sept. 8-11, 2013, pp. 13-18.

[12] D. Kapetanovic, A. Al-Nahari, A. Stojanovic, and Fredrik Rusek, "Detection of Active Eavesdroppers in Massive MIMO," IEEE Personal, Indoor and Mobile Radio Communications (PIMRC), Washington DC, Sept. 2-5, 2014.

[13] R. Di Taranto, S. Muppirisetty, R. Raulefs, D. T. M. Slock, T. Svensson, and H. Wymeersch, "Location-aware communications for 5G networks,“ IEEE Signal Processing Magazine, pp. 102-112, vol. 31, no. 6, Nov., 2014.

[14] J. Flordelis, X. Gao, G. Dahman, F. Rusek, O. Edfors and F. Tufvesson, "Spatial separation of closely-spaced users in measured massive multi-user MIMO channels“ Accepted for publication in IEEE International Conference on Communications (ICC), London, UK, 2015.

[15] N. Nguyen, G. Zheng, Z. Han and R. Zheng, "Device fingerprinting to enhance wireless security using nonparametric Bayesian method," IEEE Annual IEEE Conference on Computer Communications (INFOCOM), Shanghai, April 2011. 

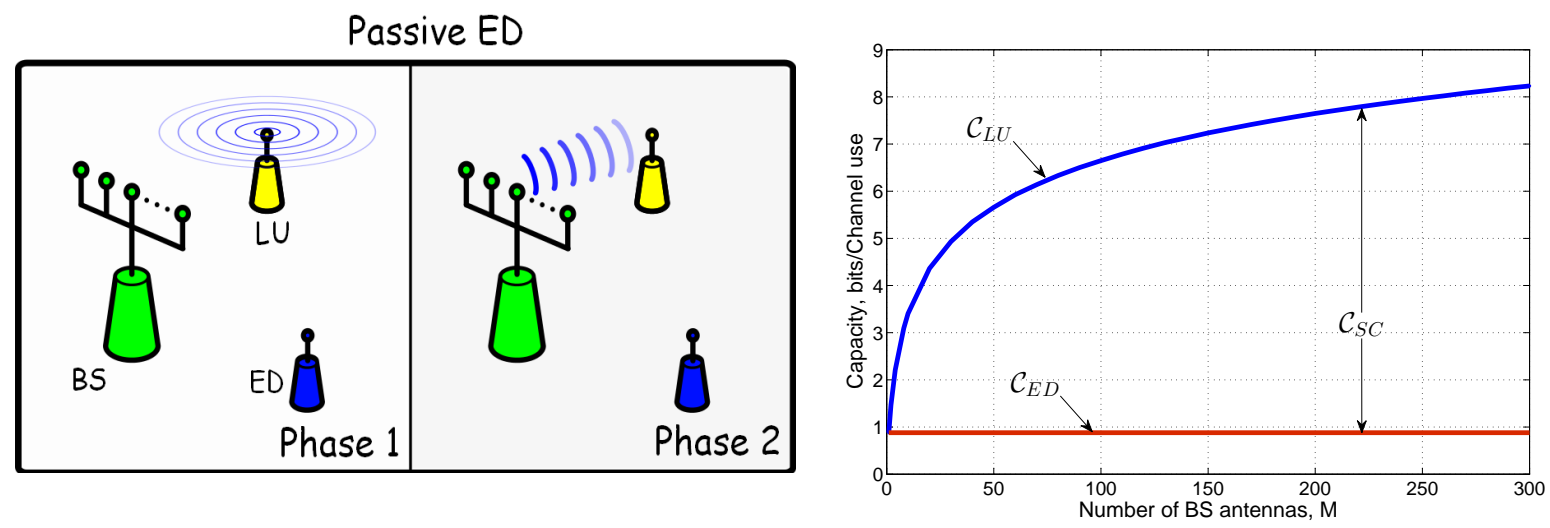

Fig. 1: Left: A single-antenna passive ED in a single cell containing a single-antenna LU. Right: Example of secrecy capacity (length of the vertical line). The ED's capacity $\mathcal{C}_{\text {ED }}$ becomes independent of $M$. The secrecy capacity increases with $M$. 

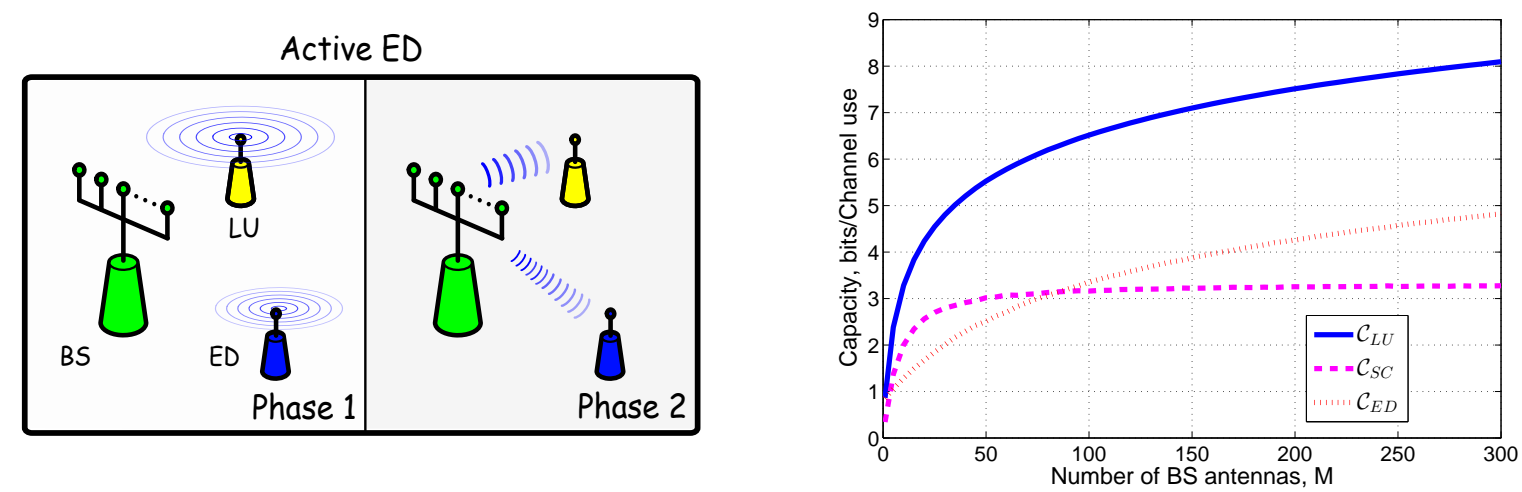

Fig. 2: Left: Active attack on the channel estimation. Right: The resulting channel capacities and the secrecy capacity. The ED's training power is $10 \mathrm{~dB}$ weaker than the LU's. 

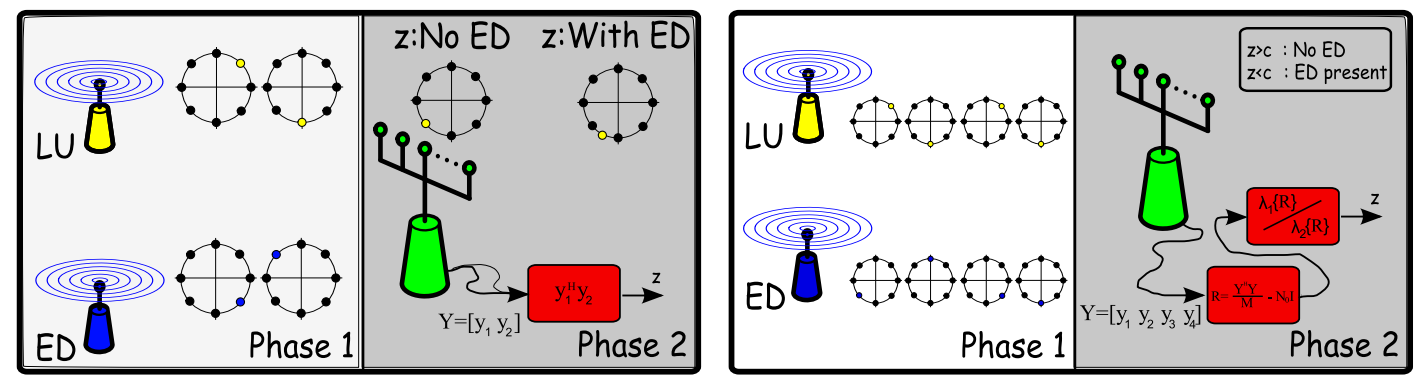

Fig. 3: Left: Detection scheme 1a. LU first transmits two random PSK symbols. After processing at the BS side, the correlation of the two received signals should (roughly) be a valid PSK symbol if there is no active ED present. Notice that case 3 is not covered in the figure. Right: Detection scheme 1b. LU transmits (in this case) four random PSK symbols. The BS constructs a correlation matrix, and performs a test based on the ratio of the two largest eigenvalues of the matrix. 


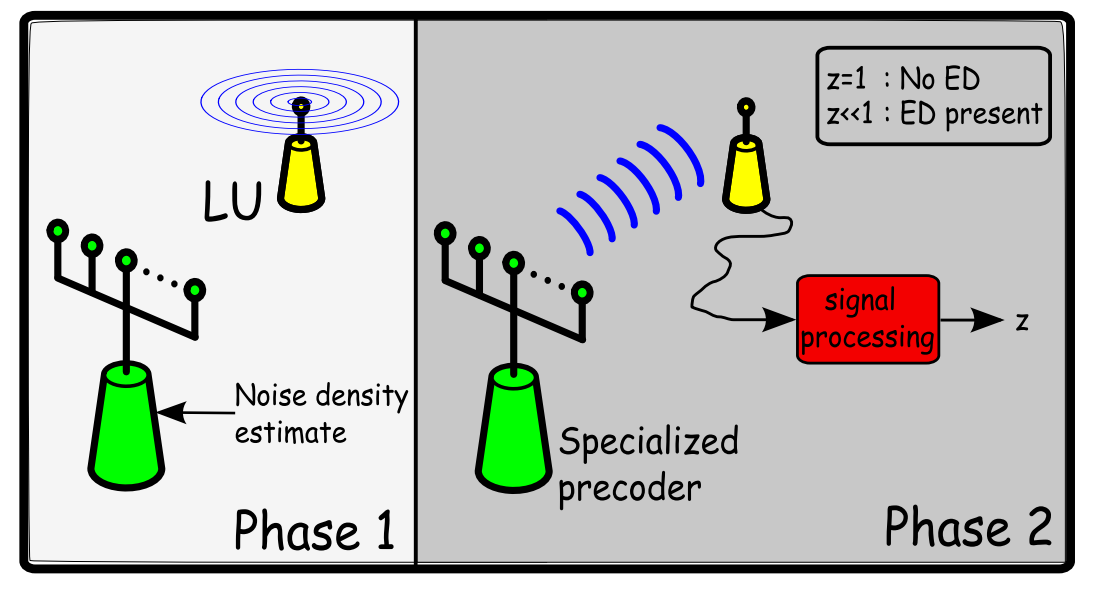

Fig. 4: Overview of Detection scheme 2. In phase 1, the BS performs channel estimation. In the next phase, the BS applies a specialized beamformer (see [12]) which ensures that the received signal at the LU after processing becomes 1. If a smaller quantity is observed, there is an active ED present. 


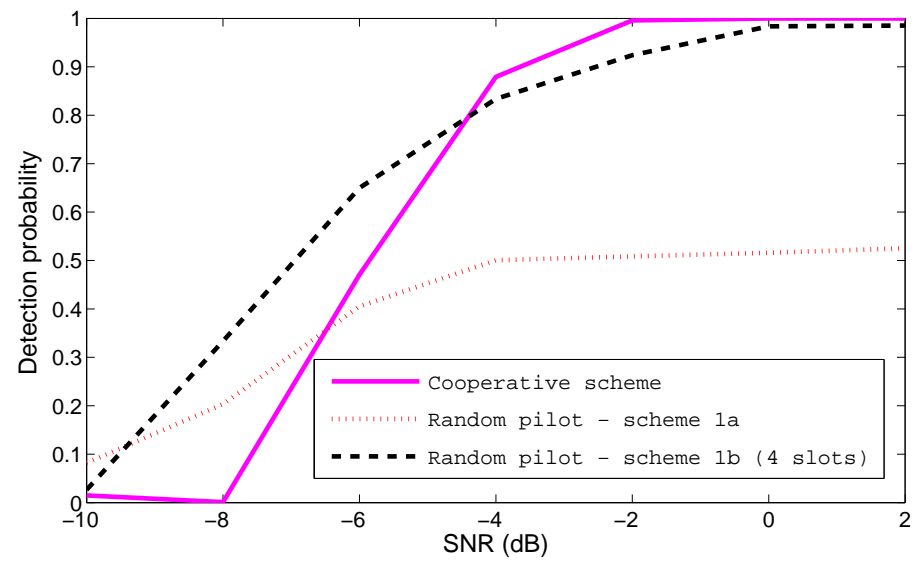

Fig. 5: Comparison of the discussed detection schemes. The false alarm probability is $1 \%$ in all schemes. The random pilot schemes use random QPSK pilots. 